EPJ Web of Conferences 75, 05015 (2014)

DOI: $10.1051 /$ epjconf/20147505015

(c) Owned by the authors, published by EDP Sciences, 2014

\title{
Study of Magnetic Nanocomposites by NMR and Bulk Magnetization Techniques
}

\author{
V. Matveev ${ }^{1,2, a}$, V. Ryzhov ${ }^{3}$, A. Lashkul ${ }^{1}$, A. Mazur ${ }^{4}$, V. Semenov ${ }^{2}$, and E. Lähderanta ${ }^{1}$ \\ ${ }^{1}$ Lappeenranta University of Technology, 53851, Lappeenranta, Finland \\ ${ }^{2}$ Saint-Petersburg State University, 198504 Saint Petersburg, Russia \\ ${ }^{3}$ Petersburg Nuclear Physics Institute, NRC « Kurchatov Institute », Russia \\ ${ }^{4}$ Physico-Technical Institute, NAN of Ukraine, Donetsk, Ukraine
}

\begin{abstract}
Magnetic nanocomposites possess complex and nonuniform magnetic structure. As a result it is necessary to use different physical methods to describe their properties. In this work we have applied a combination of micro and macro approaches to understand more deeply magnetic properties of some cobaltcontaining nanocomposites. Testing of magnetic structure of the samples at molecular level was done with NMR and Mössbauer techniques whereas static (SQUID) and dynamic magnetic $\left(\mathrm{M}_{2}\right.$, see below) measurements - at macro level.
\end{abstract}

\section{Introduction}

Magnetic nanocomposites have aroused significant scientific and technological interest due to their unique properties and wide area of possible applications, see e.g. [1] and references within. In the case of metal nanoparticles synthesis, the major problem is instability of oxidation of the particles and a necessity to control the size and shape of them. Hence, investigation of the physical properties such as magnetic behavior etc. in systems with reduced dimensionality requires an application of as wide set of the techniques as possible. In our case we tested two magnetic nanomaterials, based on cobalt-containing clusters fixed on a surface of nanoTeflon (PTFE) granules. The first composite (further "Co") contained cobalt clusters while the second composite (further "FeCo") included those of bimetallic iron-cobalt clusters. To clarify magnetic state of the samples as a whole (bulk level) we employed measurements of static magnetization with SQUID magnetometry, and registration of the second harmonic of magnetization of longitudinal nonlinear response to a weak $a c$ field ( $M_{2}$-response, see below). To test local magnetic state of cobalt and iron in the samples under study we used NMR and Mössbauer techniques.

\section{Experimental details}

Nanocomposites under study were prepared by the method of thermal decomposition as described earlier, see [2] and references within. An average diameter of the particles was about $10 \mathrm{~nm}$, a total cobalt concentration was equal to 5 mass. $\%$ in both samples, and iron concentration was 5 mass. $\%$ in the "FeCo" samples.

A dependence of magnetization $M$ against temperature was measured with a SQUID magnetometer in a constant magnetic field in a range $1 \mathrm{mT}-6 \mathrm{~T}$ when heating the sample preliminary cooled from $300 \mathrm{~K}$ down to $3 \mathrm{~K}$ in zero field (zero-field cooled magnetization, $M_{\mathrm{ZFC}}$ ) or in the applied field (field-cooled magnetization, $M_{\mathrm{FC}}$ ). Thermo remanent magnetization (TRM) was investigated in zero magnetic field when heating after cooling the sample from $300 \mathrm{~K}$ to $3 \mathrm{~K}$ in a field. The magnetization data below are presented after subtraction of the diamagnetic contribution.

${ }^{59}$ Co NMR signals were detected by spin-echo method using a home-made noncoherent pulse spectrometer with a frequency sweep and boxcar detector signal averaging. The NMR spectra were recorded by measuring the integrated intensity of the spin-echo versus frequency after a two-pulse sequence $\tau^{-} \tau_{1-2}-\tau$, where $\tau_{1-2}=7.2 \mu \mathrm{s}$ is the interval between the excitation pulses with duration $\tau=0.6 \mu \mathrm{s}$.

${ }^{57} \mathrm{Fe}$ Mössbauer spectra were recorded in transmission geometry by a conventional constant acceleration apparatus WISSEL with a ${ }^{57} \mathrm{Co} / \mathrm{Rh}$ source. The spectra parameters were refined by MOSFIT fitting software. The velocity scale was calibrated with a standard thin $\alpha$-iron foil at room temperature.

The measurements of the second harmonic of magnetization, $M_{2}$, of the longitudinal nonlinear response (NLR) were performed in the parallel steady and alternating magnetic fields $H(t)=H+h \sin \omega t(h \approx 14.3$ Oe, $f=\omega / 2 \pi \approx 15.7 \mathrm{MHz}$ ) under condition $M_{2} \propto h^{2}$. The

\footnotetext{
a Corresponding author: vmatveev@nmr.phys.spbu.ru
} 


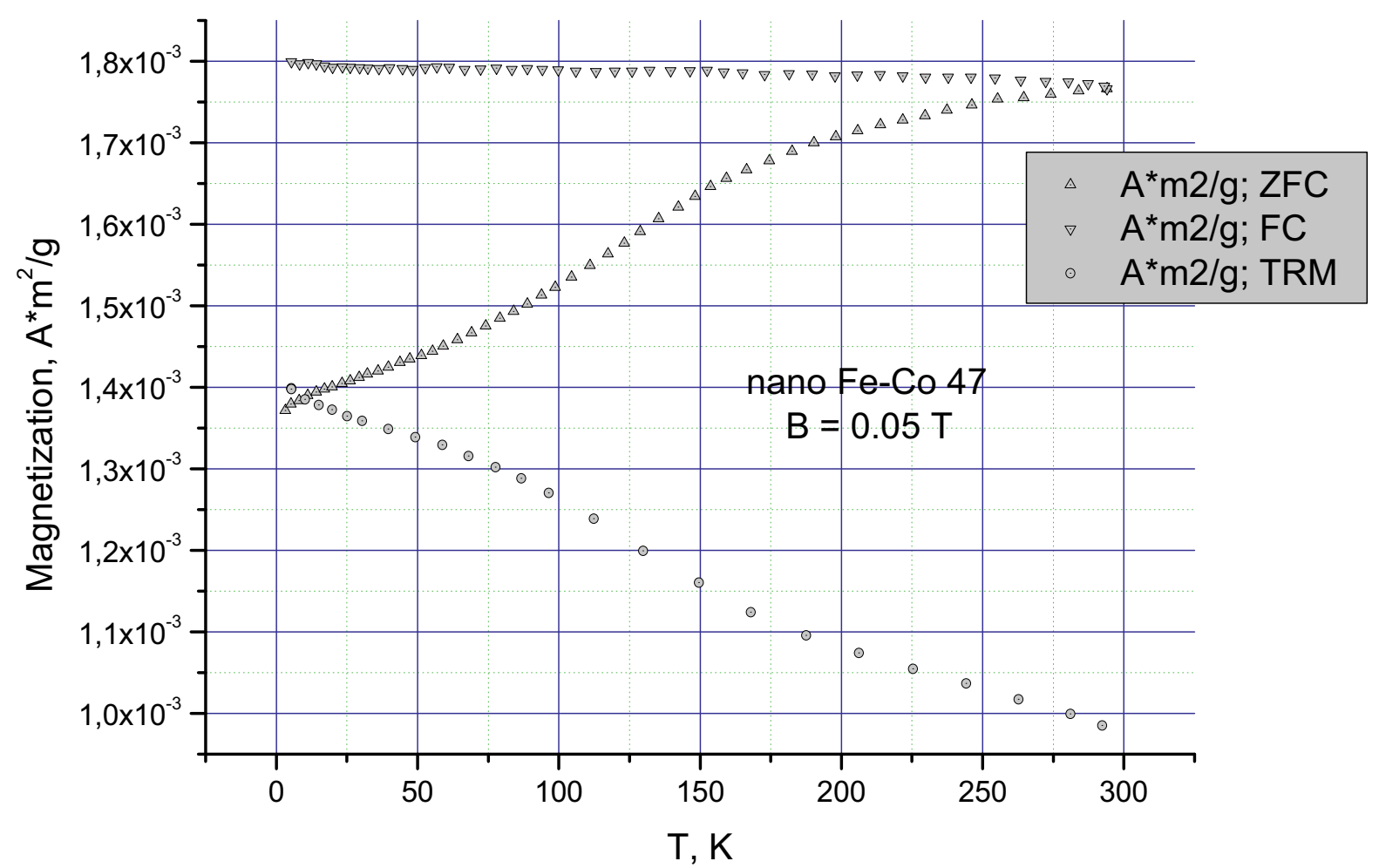

Fig. 1. Magnetization vs. temperature for the "FeCo" nanocomposite at $50 \mathrm{mT}$ applied field.

last allows one to analyze the results in the framework of perturbation theory. $\operatorname{Re} M_{2}$ and $\operatorname{Im} M_{2}$ phase components of $M_{2}$ response were simultaneously recorded as the functions of $H$ at the various sample temperatures in the range from $160 \mathrm{~K}$ up to $430 \mathrm{~K}$. The field $H$ was scanned symmetrically relative to the point $H=0$ with frequency $F_{\text {sc }}$ for control of a field hysteresis in the signal, which appearance due to symmetry properties of $M_{2}$ indicates arising the spontaneous ferromagnetic moment in a sample. The method is very sensitive to appearance of a ferromagnetic (F) component in magnetization owing to its noticeable nonlinearity in weak magnetic fields. The amplitude of $H$-scan was $300 \mathrm{Oe}$ and $F_{\text {sc }}$ could be changed over the range $0.01-10 \mathrm{~Hz}$. An installation and a method of separation of the $M_{2}$-phase components have been described previously [3]. Sensitivity of these measurements was about $10^{-9} \mathrm{emu}$.

\section{Results and discussion}

\subsection{SQUID data}

First, we tested bulk magnetic properties of the samples using SQUID measurements as well-known technique for investigation of various magnets. Some data for the "FeCo" sample obtained at low magnetic field are shown in Fig. 1. Presence of TRM indicates a magnetic-ordering state in both our samples up to temperatures above 300 $\mathrm{K}$. Small increasing of $M_{\mathrm{FC}}(T)$ at cooling as well as large deviation of $M_{\mathrm{FC}}(T)$ and $M_{\mathrm{ZFC}}(T)$ suggest a clustered state of the samples, including magnetically ordered non- interacting (in the first approximation) clusters in accordance with the known configuration of the samples consisting of metal nanoparticles placed on a surface of PTFE granules. At the same time the dependence of magnetization on temperature at $B=6 \mathrm{~T}$ does not show any difference between $\mathrm{ZFC}$ and FC curves. This evidences that the Zeeman energy essentially exceeds an anisotropy one in the field used.

The total magnetization of the "FeCo" sample may include contributions not only from the ferromagnetic metallic cobalt but from a ferromagnetic metallic iron as well. The $M_{s}$ at $6 \mathrm{~T}$ was equal to $\sim 4.5 / 4.0 * 10^{-3} \mathrm{~A} * \mathrm{~m}^{2} / \mathrm{g}$, however it was impossible to divide this value between cobalt and iron contributions using only SQUID data. Therefore we employ NMR spectroscopy in the NMR-inmagnetics variant and Mössbauer spectroscopy in order to test the samples magnetic properties at local level. ${ }^{59} \mathrm{Co}$ NMR in zero magnetic field (also known as "NMR-inmagnets" or "spin echo" technique) allows one to check if the metallic cobalt exists in the nanocomposites, whereas ${ }^{57} \mathrm{Fe}$ Mössbauer spectrum can reveal an information about a status/phases of iron in the "FeCo" sample.

\subsection{NMR and Mössbauer data}

A spectrum of the "Co" sample was already detected previously at $77 \mathrm{~K}$ [2] and indicated the main signal near $226 \mathrm{MHz}$ corresponded to metallic cobalt in the form of hcp phase. A spectrum of the "FeCo" sample is displayed in figure 2, and shows metallic cobalt as well, mostly in the hcp phase also.

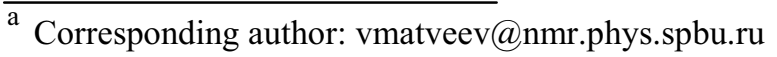


${ }^{57} \mathrm{Fe}$ Mossbauer spectrum of the "FeCo" sample displayed in figure 3 shows explicitly that iron is present only in a form of oxides. No ferromagnetic metallic-iron sextet was observed at either $77 \mathrm{~K}$ or room temperature.

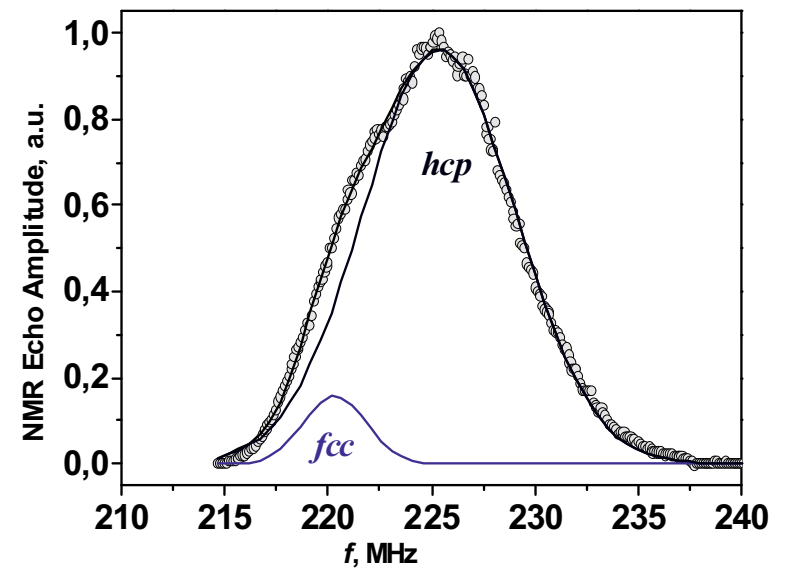

Fig. 2. ${ }^{59} \mathrm{Co}$ NMR spin-echo spectrum of the FeCo nanoparticles at $77 \mathrm{~K}$. Points correspond to experimental data, solid lines - to full fit as well as hep and fec components. Ratio of hep to fcc phase is ca. 9.0/0.60.

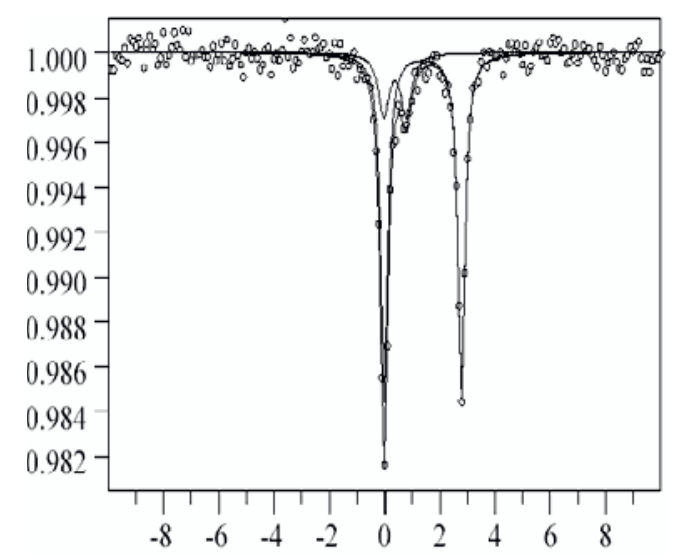

Fig. 3. ${ }^{57} \mathrm{Fe}$ Mossbauer spectrum of FeCo nanoparticles on UPFE surface, $\mathrm{T}=298 \mathrm{~K}$. Larger QS (quadrupolar splitting) component corresponds to $\boldsymbol{F e}^{\boldsymbol{2}^{+}}$, and smaller QS one - to $\boldsymbol{F} \boldsymbol{e}^{3+}$.

On the contrary, two different iron oxides, of $\boldsymbol{F e}^{2+}$ and of $\mathrm{Fe}^{3+}$ have been detected, their relative amounts being equal to $79.4 \%$ and $20.6 \%$ accordingly. Thus, combining NMR and Mössbauer data it is possible to picture "FeCo" cluster in the form of a core-shell nanoparticle with a core of metallic cobalt and with the iron oxides making an external "patch" layer of the cluster, see figure 4.

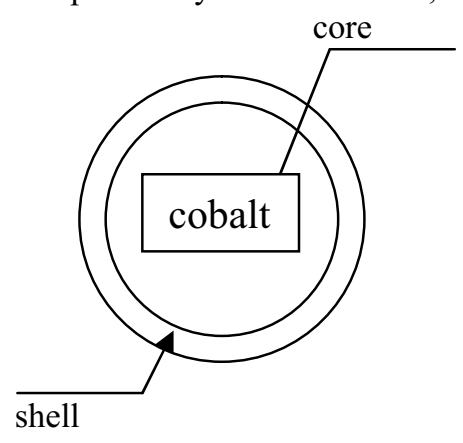

Fig. 4. Schematic structure of core-shell FeCo nanoparticle. See details in the text.
As it was mentioned above metallic core in cobalt nanoparticles is commonly covered by cobalt-oxide shell. The formation of this layer is due to process of cobalt oxidation during the preparation process. NMR can not detect cobalt oxides as their frequencies are far from available range of the device used in this work. Therefore we tried to use a relatively new method - registration of the second harmonic of magnetization of longitudinal nonlinear response to a weak ac field $\left(M_{2}\right)$ - in order to try to obtain some additional information concerning cobalt clusters. We applied this technique only for "Co" sample due to its simpler composition comparing with "FeCo" one.

\subsection{Second harmonic of magnetization data}

In the simple case of uniaxial anisotropy a magnetic moment of nanoparticle (that is the case for Co one [2]) is aligned along this axis and without an external magnetic field both directions of magnetization in the anisotropy axis are equivalent, while at presence of field one of them is preferred. After the field switching off the magnetization should mount the energy barrier $E_{b}=K V$ ( $K$ is an anisotropy constant, and $V$ is a volume of particle) to rotate back to the state defined by anisotropy, which takes a time. The relaxation time of this process $\tau$ is given by $1 / \tau=\Gamma=f_{0} \exp \left(-E_{b} / k T\right)$ [4], where the Larmor frequency $f_{0}$ is of the order of $10^{9} \mathrm{~s}^{-1}$. Because the particle volume $V$ and temperature $T$ are both in the exponent, the value of $\tau$ is strongly dependent on them. The probability that the magnetization remains in its original position during time $t$ after switching off the external field can be written as $P(t)=\exp (-t / \tau)$, that gives for retentivity $M_{\text {ret }}(t) / M_{0}=\exp (-t / \tau)$. Thus, if the registration time is less than $\tau$, the $M_{\text {ret }}$ will be detected. In our case of the periodic $H$-scan and synchronous detection of a response, $H$-hysteresis will be observed in $M_{2}(H)$ response, when a period of $H$-scan $1 / F_{\mathrm{sc}} \leq \tau=1 / \Gamma$. For standard measurements of $M_{\text {ret }}$ the time $\tau^{*}=100 \mathrm{~s}$ is normally used that gives the limit of SPM behavior $K_{l} V=25 k T$. From this relation for the ensemble of identical particles the blocking temperature $T_{\mathrm{B}}$ iis introduced, above which the behavior of the system will be superparamagnetic without the field hysteresis in $M_{2}(H)$-response. It is given by expression $T_{\mathrm{B}}=K V / 25 k$ [4]. In blocking regime below $T_{\mathrm{B}}$ the field hysteresis depending on $F_{\mathrm{sc}}$ should be observed in $M_{2}(H)$. To clarify whether "Co" composite sample provides a typical for an ensemble of nanoparticles $M_{2}(H)$ response with an extremum in a weak steady field $H$ and field hysteresis, which depends on $T$ and $F_{\text {sc }}$, we obtained the corresponding dependences. Figure 5 presents the phase components $\operatorname{Re} M_{2}(H)$ and $\operatorname{Im} M_{2}(H)$ recorded versus $H$ at several magnitudes of $F_{\text {sc }}$ for "Co" composite. As one can see from the figure, the response includes two types of signals. The larger signal, contributed mainly to $\operatorname{Re} M_{2}(H)$, has no extremum in weak $H$ and exhibits opposite (positive) sign relative to usual one (negative) of a signal from magnetic nanoparticles as well as $H$ hysteresis which is not change at decreasing $F_{\text {sc }}$ (figure $5 a)$. The weak signal in $\operatorname{Im} M_{2}(H)$ exhibits an extremum in 
a weak field $H$ and reveals the strong dependence of $H$ hysteresis on $F_{\mathrm{sc}}$ (figure $5 \mathrm{~b}$ ). The contribution to the $\operatorname{Re} M_{2}(H)$ from this signal is hardly seen in Fig.5a. The $M_{2}$ response reveals the similar features at heating of the sample from 160 up to $430 \mathrm{~K}$, specifically the decreasing/ invariability of $H$-hysteresis in $\operatorname{Im} M_{2} / \operatorname{Re} M_{2}$ accordingly is found (are not presented). As it was discussed above the Co cluster probably consists of $\mathrm{F}$ metal Co core and an oxide shell with usual for Co oxides antiferromagnetic arrangement (AF). The availability of structure defects on/near surface of clusters implies a presence of $\mathrm{F}$ component in the AF response of the shell to external field. This suggests observation of $M_{2}(H)$ response without extremum in a weak $H$ from the AF shell (since large $H$ is necessary to decouple its magnetic sublattices) and a characteristic signal with the weak $H$ extremum and dependence on $F_{\text {ac }}$ from $\mathrm{F}$ core in agreement with $M_{2}$ data. In the shell a relative amount of Co atoms can be essentially larger than in core in accordance with the amplitudes of these signals ratio in $\operatorname{Re} M_{2}(H)$ (figure $5 \mathrm{a}$ ). The signal from the $\mathrm{F}$ core is better observed in $\operatorname{Im} M_{2}(H)$ owing to more effective magnetic relaxation of AF shell $\left(\operatorname{Im} M_{2} \propto(\omega / \Gamma) \operatorname{Re} M_{2}\right.$ in first approximation without taking into account an effect of field on relaxation). So the latter gives a small contribution to $\operatorname{Im} M_{2}$. A signal with the positive sign in $\operatorname{Re} M_{2}$ component was earlier observed in $M_{2}$ response of $\mathrm{AF} \mathrm{La}_{2} \mathrm{CuO}_{4+\mathrm{x}}$, possessing by a weak ferromagnetism [5] that supports our assumption.
Thus, the $M_{2}$ data suggest a presence of a core-shell structure of Co nanoparticles in "Co" composite similar to that of $\mathrm{FeCo}$ clusters in "FeCo" one. The core is formed by $\mathrm{F}$ ordered metallic $\mathrm{Co}$ and $\mathrm{AF}$ oxide shell arises due to oxidation of $\mathrm{Co}$ at sample preparation and exhibits a considerable $\mathrm{F}$ component owing to structure defects near particle surface.

\section{References}

1. T. Wen, K.M. Krishnan, J. Phys. D: Appl. Phys., 44, 393001 (2011)

2. V.V. Matveev, D.A. Baranov, G.Yu. Yurkov, N.G. Akatiev, I.P. Dotsenko, S.P. Gubin, J. Chem. Phys. Lett., 422, 402 (2006)

3. V.A. Ryzhov, I.I. Larionov, V.N. Fomichev, Zh. Tekh. Fiz. 66 (1996) 183 [Sov. Phys. Tech. Phys. 41 (1996) 620]

4. B.D. Gullity, C.D. Graham. In: Introduction to Magnetic Materials (IEEE Press, WILEY, 2009), pp. 383-387

5. A.V. Lazuta, V.A. Ryzhov, I.I. Larionov, T.I. Arbuzova, Physica C, 295, 22 (1998)
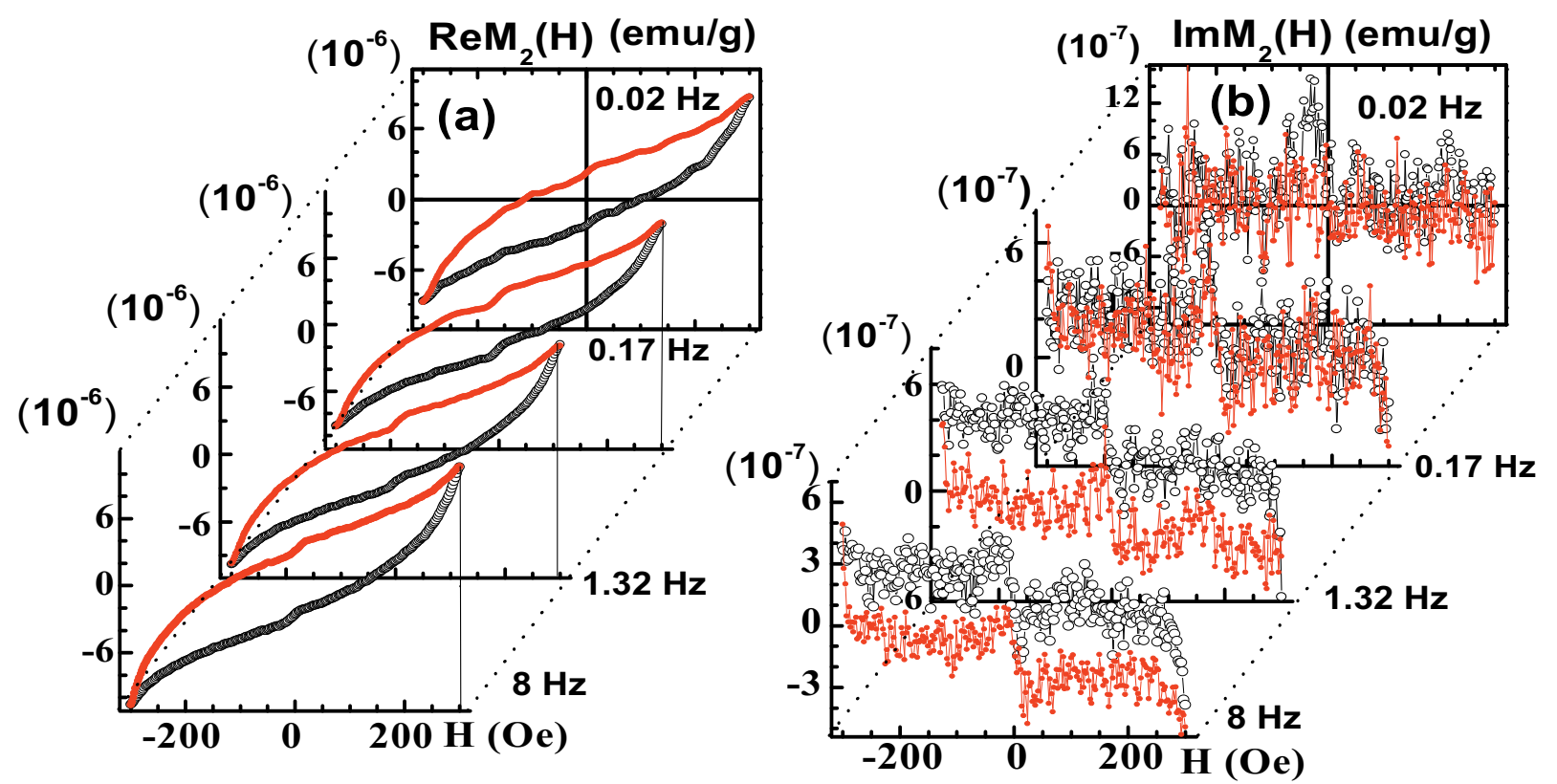

Fig. 5. $\operatorname{Re} M_{2}(H)$, panel (a) and $\operatorname{Im} M_{2}(H)$, panel (b) dependences obtained at $T \approx 416 \mathrm{~K}$ for "Co" composite at different $F_{\text {sc. }}$. Solid and open symbols are used for the curves recorded at direct and reverse $H$-scans, respectively. See details in the text. 